\title{
Efetividade da infiltração de corticosteroides no tratamento de pacientes com tenossinovite estenosante de De Quervain: resultado terapêutico
}

Effectiveness of corticosteroid injections for treatment of patients with Quervain's tenosynovitis: therapeutic outcome Efectividad de inyección de corticosteroides para el tratamiento de pacientes con tenosinovitis de De Quervain: resultado terapéutico

Bruno Moraes VASCONCELOS

Luis Guilherme Rosifini ALVES REZENDE Henrique Gontijo CHAMON ${ }^{3}$

Luiz Henrique VILELA ${ }^{4}$

${ }^{I}$ Especialista em Ortopedia e Traumatologia pelo Hospital Márcio Cunha, Fundação São Francisco Xaxier (FSFX)Ipatinga-MG, Brasil Residente, Cirurgia da Mão, Hospital das Clínicas da Faculdade de Medicina de Ribeirão Preto, Universidade de São Paulo (USP) 14049-900 Ribeirão Preto-SP, Brasil ${ }^{2}$ Especialista em Ortopedia e Traumatologia pelo Hospital das Clínicas da Faculdade de Medicina de Ribeirão Preto da Universidade de São Paulo (USP); Residente, Cirurgia da Mão, Hospital das Clínicas da Faculdade de Medicina de Ribeirão Preto da Universidade de São Paulo (USP);

Mestrando Programa de Pós-Graduação Ciências da Saúde Aplicadas ao Aparelho Locomotor/Faculdade de Medicina de Ribeirão Preto, Universidade de São Paulo (USP) 14049-900 Ribeirão Preto-SP, Brasil

${ }^{3}$ Especialista em Cirurgia de Mão pela Casa de Saúde Santa Izabel (CSSI), Fundação Hospitalar do Estado de Minas Gerais (FHEMIG), 32850-000 Betim-MG, Brasil ${ }^{4}$ Especialista em Ortopedia e Traumatologia pelo Hospital das Clínicas da Faculdade de Medicina de Ribeirão Preto da Universidade de São Paulo (USP) 14049-900 Ribeirão Preto-SP, Brasil

\section{Resumo}

Introdução: A tenossinovite estenosante de De Quervain, doença inflamatória mais comum do punho, de maior prevalência no sexo feminino e na faixa etária de 30 a 50 anos, caracteriza-se pelo envolvimento da bainha sinovial do primeiro compartimento extensor do punho, que inclui os tendões abdutor longo do polegar (ALP) e extensor curto do polegar (ECP). Apesar da etiologia indefinida, atribui-se caráter multifatorial como traumas, sobrecarga de exercícios repetitivos com punho e mão, alterações metabólicas, reumáticas e variações anatômicas, como a presença de dois ou mais tendões do ALP e ECP e septo no túnel osteofibroso. Dentre as alternativas terapêuticas encontra-se a infiltração com corticosteroides. Material e Método: Foi avaliado o resultado terapêutico em 15 pacientes submetidos à infiltração local de corticosteroide através dos questionários DASH, PRWE e Escala Visual da Dor. Resultados: observou-se melhora significativa dos pacientes avaliados. Conclusão: o tratamento com corticosteroides é eficaz e seguro.

Descritores: Doença de De Quervain; Tenossinovite; Corticosteroides.

\section{Abstract}

Introduction: Quervain's tenosynovitis, the most common inflammatory wrist disease, most prevalent in females and in the age group of 30 to 50 years, is characterized by the involvement of the synovial sheath of the first extensor compartment of the wrist, which includes the thumb long abductor tendons (ALP) and short thumb extensor (ECP) tendons. Despite the undefined etiology, multifactorial character is attributed as trauma, overload of repetitive exercises with wrist and hand, metabolic, rheumatic alterations and anatomical variations, such as the presence of two or more ALP and ECP tendons and osteofibrosal tunnel septum. Among the therapeutic alternatives is infiltration with corticosteroids. Material and Method: The therapeutic outcome was evaluated in 15 patients who underwent local corticosteroid infiltration using the DASH and PRWE questionnaires and Visual Analogue Scale. Results: Significant improvement was observed in the evaluated patients. Conclusion: Corticosteroid treatment is effective and safe.

Descriptors: De Quervain Disease; Tenosynovitis; Adrenal Cortex Hormones.

\section{Resumen}

Introducción: la tenosinovitis de De Quervain, la enfermedad inflamatoria de la muñeca más común, más prevalente en mujeres y en el grupo de edad de 30 a 50 años, se caracteriza por la afectación de la vaina sinovial del primer compartimento extensor de la muñeca, que incluye el tendones abductores largos del pulgar (ALP) y tendones extensores del pulgar cortos (ECP). A pesar de la etiología indefinida, el carácter multifactorial se atribuye a trauma, sobrecarga de ejercicios repetitivos con muñeca y mano, alteraciones metabólicas, reumáticas y variaciones anatómicas, como la presencia de dos o más tendones ALP y ECP y tabique osteofibrosal. Entre las alternativas terapéuticas se encuentra la infiltración con corticosteroides. Material y método: El resultado terapéutico se evaluó en 15 pacientes que se sometieron a infiltración local de corticosteroides utilizando los cuestionarios DASH y PRWE y escala visual analógica del dolor. Resultados: se observó una mejoría significativa en los pacientes evaluados. Conclusión: el tratamiento con corticosteroides es efectivo y seguro.

Descriptores: Tenosinovitis; Enfermedad de De Quervain; Corticoesteroides.

INTRODUÇÃO

A tenossinovite estenosante De Quervain é uma afecção inflamatória da bainha sinovial do primeiro compartimento extensor do punho, afetando os tendões abdutor longo do polegar (ALP) e extensor curto do polegar (ECP) ${ }^{1}$. É uma das afecções mais comuns do punho com prevalência de $0,5 \% \mathrm{em}$ homens e 1,3\% em mulheres, comumente entre os 30 a 50 anos de idade, com maior incidência em mulheres jovens no período gestacional ou lactação ${ }^{1-4}$. Além disso, possui sua incidência correlacionada com a quantidade da força necessária para os movimentos no punho e das mãos durante o trabalho, atém de movimentos de repetição ${ }^{4,5}$. Apesar da etiologia indefinida, atribui-se caráter multifatorial como traumas, sobrecarga de exercícios repetitivos com punho e mão, alterações metabólicas, reumáticas e variações anatômicas, como a presença de dois ou mais tendões do ALP e ECP e septo no túnel osteofibroso ${ }^{3,4,6,7}$.

O diagnóstico é baseado na história clínica e exame físico, caracteristicamente pela existência de dor insidiosa na face radial do punho, agravada por movimentos de abdução e extensão do polegar com o punho em extensão e desvio ulnar ${ }^{3-5}$.

Seu tratamento consiste na remoção do agente predisponente, associados a medidas conservadoras e, em caso de persistência, institui-se o tratamento cirúrgico, que consiste na liberação dos tendões do primeiro compartimento extensor do punho $^{3,4,7}$. Diversas formas de tratamento conservador foram relatadas incluindo acupuntura, terapia da mão, fisioterapia, crioterapia, ultrassom, laser, eletroterapia, uso de anti-inflamatórios não esteroidais, imobilização com órteses e infiltração 
local de corticosteroide ${ }^{2,3,8-13}$. As infiltrações de corticosteroides são consideradas são as principais opções na abordagem conservadora do tratamento da doença De Quervain, considerando que em alguns estudos são frequentemente citadas como melhor prática terapêutica ${ }^{2,3,8,11,12 \text {, }}$

O propósito deste trabalho foi avaliar os resultados terapêuticos nos pacientes submetidos à infiltração de corticosteróide no tratamento da tenossinovite de De Quervain através do questionário Quick DASH, PRWE e Escala Visual da Dor.

\section{MATERIAL E MÉTODO}

Foram avaliados 15 pacientes portadores de tenossinovite de De Quervain isolada, diagnosticados previamente no período de um ano entre os meses de junho de 2015 e junho de 2016 e submetidos ao tratamento conservador com infiltração de corticosteroides.

Todos os participantes eram do sexo feminino, com idades compreendidas entre 31 anos e 52 anos. Do total de entrevistadas, oito $(53,3 \%)$ eram gestantes ou lactantes; sete pacientes $(46,7 \%)$ foram acometidas pela doença no punho esquerdo, duas $(13,3 \%)$ no punho direito, e seis $(40 \%)$ em ambos os punhos. Todas as pacientes avaliadas foram submetidas à infiltração de corticosteroide, sendo que o número infiltrações realizadas variou entre uma e quatro, dependendo da necessidade apresentada por cada paciente. Os critérios de exclusão considerados foram quaisquer pacientes com outras comorbidades, como hipertensão e tabagismo.

A infiltração foi realizada após infiltração de anestesia local (lidocaína) e infiltração de $1 \mathrm{ml}$ do medicamento acetato de metilprednisolona em associação com $1 \mathrm{ml}$ do medicamento neocaína em túnel osteofibroso do primeiro compartimento extensor do punho.

As pacientes receberam avaliação clínica e funcional, através de medidas da amplitude de movimento do punho em Graus, usando o instrumento goniômetro e também foi avaliada a força de preensão usando um dinamômetro em kgf. Os dados foram comparados ao membro contralateral sem lesão.

Os questionários Quick DASH e PRWE foram aplicados a todos os participantes pelo pesquisador e residente de ortopedia, levando-se em consideração os diferentes níveis de escolaridade e socioculturais dos pacientes.

O questionário Quick DASH (Disfunção Braço, Ombro e Mão) apresenta 11 questões que busca coletar informações a respeito do grau de dificuldade no desempenho de atividades diárias, sintomas de dor, fraqueza, rigidez e desconforto pulso afetado. As questões são pontuadas entre um e cinco, tendo seu escore final compreendido entre zero (sem disfunção) e 100 pontos (disfunção grave). O escore total é calculado a partir da somatória da pontuação de todas as questões, com o resultado dividido por 11 (número completo de respostas), menos 1 (um) e multiplicado por 25. Quanto menor o escore obtido, menor o grau de disfunção verificado e, consequentemente, quanto maior o escore registrado, maior o grau de disfunção identificado.

O questionário PRWE (Avaliação Nominal do Punho do Paciente) se propõe a coletar informações para que se compreenda o grau de dificuldade que o paciente teve com o punho afetado na semana anterior, bem como o grau de dor prevalecente. São apresentadas 15 questões agrupadas em duas categorias: dor e função, que são pontuadas de zero a dez, considerando zero como ausência de dor ou ausência de dificuldade para realizar a tarefa e 10 como pior dor sentida ou impossibilidade de realizar a tarefa apresentada. Esse questionário tem sua pontuação calculada pela somatória simples e direta de todas as questões apresentadas em cada etapa, com o resultado dividido pelo total possível e multiplicado por 100. O resultado final de cada etapa é compreendido numa escala numérica que varia entre zero e dez. Quanto menor o resultado obtido, menor o grau de dificuldade na realização das atividades (maior capacidade) e menor o índice de dor; quanto maior o resultado, maior a dificuldade na realização das atividades (menor capacidade) e maior o índice de dor apresentado pelo paciente. A pontuação total do questionário, considerando a soma das duas etapas, varia entre zero e vinte, obedecendo ao mesmo critério de quanto menor, maior capacidade e menor índice de dor; e quanto maior, menor capacidade e maior índice de dor.

Os dois questionários foram aplicados aos pacientes no período compreendido entre os dias 15 a 30 de junho de 2017, perfazendo um período mínimo de um ano após a submissão dos pacientes ao tratamento por infiltração de corticosteroide.

\section{RESULTADOS}

\section{- Avaliação clínica e funcional}

Preliminarmente, as pacientes foram submetidas à avaliação clínica e funcional, incluindo amplitude de movimento do punho (desvio ulnar, desvio radial, flexão e extensão) e teste de força em ambos os punhos. Apenas uma participante (P13) não realizou a avaliação por não apresentar condições físicas em função de uma recente cirurgia realizada. Os resultados são apresentados na Tabela 1 .

\section{- Resultados e análise do questionário Quick DASH}

Os resultados verificados a partir da aplicação do questionário Quick DASH apontaram que, de forma geral, existe um grau pequeno ou moderado de disfunção em uma pequena parcela dos pacientes avaliados. Esses casos incluem apenas cinco pacientes (P4, P7, P9, P12 e P13), ressaltando um quadro geral muito positivo em relação às questões avaliadas, uma vez que a maioria dos pacientes teve pontuação zero, 
ou seja, 10 do total de 15 apresentaram ausência de disfunção em todos os quesitos pontuados. Ainda assim, o grau de disfunção apresentado pelos cinco pacientes não se mostrou preocupante, mas, ao contrário, situou-se, de forma geral, em patamares reduzidos e considerados leves, ficando evidente na variação entre 2,3 para o menor desses graus e 27,3 para o maior deles. Esse aspecto é reforçado pela média total que foi de 4,8 , indicando que, em relação às questões apresentadas pelo questionário Quick DASH, os pacientes avaliados, em geral, estão aptos a realizar as funções correspondentes. Esse cenário está refletido na Tabela 2.

Tabela 1. Resultados da avaliação clínica e funcional dos pacientes

\begin{tabular}{|c|c|c|c|c|c|c|c|c|c|c|}
\hline \multirow[t]{2}{*}{ Paciente } & \multicolumn{2}{|c|}{ Força* } & \multicolumn{2}{|c|}{$\begin{array}{l}\text { Desvio } \\
\text { Ulnar*** }\end{array}$} & \multicolumn{2}{|c|}{$\begin{array}{c}\text { Desvio } \\
\text { Radial }^{* * *}\end{array}$} & \multicolumn{2}{|c|}{ Flexão** } & \multicolumn{2}{|c|}{ Extensão } \\
\hline & D & $\mathbf{E}$ & D & $\mathbf{E}$ & D & $\mathbf{E}$ & D & $\mathbf{E}$ & D & $\mathbf{E}$ \\
\hline P1 & 18,8 & 11,8 & $28^{\circ}$ & $34^{\circ}$ & $22^{\circ}$ & $14^{\circ}$ & $30^{\circ}$ & $30^{\circ}$ & 0 & 0 \\
\hline $\mathbf{P 2}$ & 15,6 & 11,1 & $40^{\circ}$ & $36^{\circ}$ & $20^{\circ}$ & $16^{\circ}$ & $40^{\circ}$ & $40^{\circ}$ & 0 & 0 \\
\hline P3 & 37,8 & 31,6 & $46^{\circ}$ & $50^{\circ}$ & $17^{\circ}$ & $18^{\circ}$ & $55^{\circ}$ & $50^{\circ}$ & 0 & 0 \\
\hline P4 & 18,9 & 14,1 & $51^{\circ}$ & $42^{\circ}$ & $22^{\circ}$ & $20^{\circ}$ & $60^{\circ}$ & $52^{\circ}$ & 0 & 0 \\
\hline $\mathbf{P 5}_{5}$ & 34,0 & 33,1 & $48^{\circ}$ & $52^{0}$ & $20^{\circ}$ & $18^{\circ}$ & $50^{\circ}$ & $60^{\circ}$ & 0 & 0 \\
\hline P6 & 32,8 & 33,9 & $42^{\circ}$ & $52^{0}$ & $18^{\circ}$ & $20^{\circ}$ & $56^{\circ}$ & $60^{\circ}$ & 0 & 0 \\
\hline $\mathbf{P}_{7}$ & 24,8 & 21,7 & $60^{\circ}$ & $50^{\circ}$ & $20^{\circ}$ & $18^{\circ}$ & $80^{\circ}$ & $80^{\circ}$ & 0 & 0 \\
\hline P8 & 26,2 & 26,1 & $40^{\circ}$ & $52^{0}$ & $18^{\circ}$ & $20^{\circ}$ & $40^{\circ}$ & $50^{\circ}$ & 0 & 0 \\
\hline P9 & 25,1 & 23,4 & $40^{\circ}$ & $40^{\circ}$ & $30^{\circ}$ & $41^{\circ}$ & $50^{\circ}$ & $51^{\circ}$ & 0 & 0 \\
\hline P10 & 22,3 & 18,1 & $51^{\circ}$ & $50^{\circ}$ & $16^{\circ}$ & $15^{\circ}$ & $50^{\circ}$ & $51^{0}$ & 0 & 0 \\
\hline P11 & 30,0 & 26,0 & $42^{\circ}$ & $40^{\circ}$ & $30^{\circ}$ & $20^{\circ}$ & $52^{\mathrm{o}}$ & $50^{0}$ & $\mathrm{O}$ & $\mathrm{O}$ \\
\hline P12 & 23,3 & 16,4 & $42^{\circ}$ & $42^{0}$ & $22^{0}$ & $18^{\circ}$ & $42^{\circ}$ & $41^{\circ}$ & 0 & 0 \\
\hline P13 & - & - & - & - & - & - & - & - & - & - \\
\hline P14 & 25,3 & 24,9 & $36^{\circ}$ & $45^{\circ}$ & $17^{\circ}$ & $18^{\circ}$ & $42^{\circ}$ & $41^{\circ}$ & 0 & $\mathrm{O}$ \\
\hline P15 & 19,8 & 21,9 & $52^{0}$ & $54^{\circ}$ & $24^{\circ}$ & $18^{\circ}$ & $50^{\circ}$ & $50^{\circ}$ & 0 & $\mathrm{O}$ \\
\hline
\end{tabular}

Tabela 2. Pontuação dos pacientes no questionário Quick DASH

\begin{tabular}{|c|c|c|c|c|c|c|c|c|}
\hline Paciente & P1 & P2 & $\mathbf{P}_{3}$ & $\mathbf{P}_{4}$ & $P_{5}$ & P6 & $\mathbf{P}_{7}$ & P8 \\
\hline $\begin{array}{l}\text { Punho } \\
\text { Avaliado* }\end{array}$ & $\mathbf{D} / \mathbf{E}$ & $\mathbf{E}$ & $\mathbf{D} / \mathbf{E}$ & $\mathbf{E}$ & $\mathbf{E}$ & $\mathbf{E}$ & $\mathbf{D} / \mathbf{E}$ & $\mathbf{E}$ \\
\hline Pontuação & 0,0 & 0,0 & 0,0 & 20,4 & 0,0 & 0,0 & 6,8 & 0,0 \\
\hline Paciente & P9 & P10 & P11 & P12 & P13 & P14 & P15 & \multirow{2}{*}{$\begin{array}{l}\text { Média } \\
\text { Total }\end{array}$} \\
\hline $\begin{array}{l}\begin{array}{l}\text { Punho } \\
\text { Avaliado }\end{array} \\
\end{array}$ & D & D & $\mathbf{D} / \mathbf{E}$ & $\mathbf{E}$ & $\mathbf{D} / \mathbf{E}$ & $\mathbf{D} / \mathbf{E}$ & $\mathbf{E}$ & \\
\hline Pontuação & 15,9 & 0,0 & 0,0 & 27,3 & 2,3 & 0,0 & 0,0 & 4,8 \\
\hline
\end{tabular}

As atividades que apresentaram maior grau de dificuldade em sua realização, por parte dos pacientes que registraram alguma pontuação total, foram as seguintes: (1) abrir um vidro novo com tampa muito apertada: três pacientes (pouca dificuldade, dificuldade moderada e dificuldade severa); (2) carregar sacola ou maleta: quatro pacientes (pouca dificuldade, dificuldade moderada e não conseguiu fazer); (3) usar uma faca para cortar alimentos: quatro pacientes (pouca dificuldade, dificuldade moderada e dificuldade severa); e (4) realizar atividades recreativas que exigem alguma força ou impacto nos braços, ombros ou mãos: três pacientes (pouca dificuldade e dificuldade moderada). Em relação à gravidade dos sintomas, apenas dois dos cinco pacientes que pontuaram no questionário apresentaram pouca gravidade no quesito dor no braço, ombro ou mão.

\section{- Resultados e análise do questionário PRWE}

Os resultados obtidos a partir da aplicação do questionário PRWE revelaram que, das 15 pacientes avaliadas, apenas sete apresentaram algum grau de dor e/ou dificuldade de realizar as tarefas propostas, cabendo destacar que todos esses registraram graus muito leves, tanto em relação à dor quanto a respeito da incapacidade de realizar tarefas. A paciente P2 foi a que apresentou maior grau incapacidade $(6,4)$, com uma média de 4,85 na soma das duas etapas, seguida pela paciente $\mathrm{P} 4$ que registro grau de incapacidade de 5,4 e uma média nas duas etapas de 4,5; as outras cinco pacientes que pontuaram apresentaram graus de dor e de incapacidade na realização das tarefas muito baixos, sendo que a paciente P5 teve o menor grau de incapacidade $(0,2)$ e a paciente P2 o menor grau de dor $(0,3)$. De forma geral, observa-se que tanto o grau de incapacidade quanto o grau de dor se mostraram bastante reduzidos e pouco significativos nas escalas globais consideradas, aspecto reforçado pela média total de incapacidade $(1,12)$ e de dor $(0,55)$, ambas consideradas muito leves, pela média da pontuação total $(1,67)$ e ainda em razão de oito pacientes não terem apresentado nenhum grau de incapacidade ou de dor.

Considerando o período entre o tratamento realizado nos pacientes, por meio de injeção de corticosteroide, e a realização da avaliação das funções e da escala de dor, através da aplicação do questionário PRWE, destaca-se o elevado grau de recuperação de todas as pacientes, ainda que algumas tenham apresentado graus leves ou moderados de dor ou incapacidade de realização das tarefas mencionadas. Também se mostra relevante o fato de que nenhuma das entrevistadas apresentou graus severos de dor ou incapacidade na realização de qualquer uma das atividades relacionadas no questionário. Evidencia-se a relevância do tratamento realizado, bem como do período de recuperação necessário para a retomada das atividades cotidianas e laborais e para a redução e/ou eliminação da dor no local afetado pela doença (Tabela 3).

Tabela 3. Pontuação dos pacientes no questionário PRWE

\begin{tabular}{c|c|c|c|c|c}
\hline Paciente & $\begin{array}{c}\text { Punho } \\
\text { Avaliado* }\end{array}$ & Função & $\begin{array}{c}\text { Escala } \\
\text { de Dor }\end{array}$ & $\begin{array}{c}\text { Pontuação } \\
\text { Total }\end{array}$ & $\begin{array}{c}\text { Média / } \\
\text { Paciente }\end{array}$ \\
\hline P1 & D/E & 2,0 & 0,0 & 2,0 & 1,0 \\
\hline P2 & E & 0,0 & 0,3 & 0,3 & 0,15 \\
\hline P3 & D/E & 0,0 & 0,0 & 0,0 & 0,0 \\
\hline P4 & E & 5,4 & 3,6 & 9,0 & 4,5 \\
\hline P5 & E & 0,2 & 0,0 & 0,2 & 0,1 \\
\hline P6 & E & 0,0 & 0,0 & 0,0 & 0,0 \\
\hline P7 & D/E & 0,0 & 0,0 & 0,0 & 0,0 \\
\hline P8 & E & 0,0 & 0,0 & 0,0 & 0,0 \\
\hline P9 & D & 2,4 & 1,1 & 3,5 & 1,75 \\
\hline P10 & D & 0,0 & 0,0 & 0,0 & 0,0 \\
\hline P11 & D/E & 0,4 & 0,0 & 0,4 & 0,2 \\
\hline P12 & E & 6,4 & 3,3 & 9,7 & 4,85 \\
\hline P13 & D/E & 0,0 & 0,0 & 0,0 & 0,0 \\
\hline P14 & D/E & 0,0 & 0,0 & 0,0 & 0,0 \\
\hline P15 & E & 0,0 & 0,0 & 0,0 & 0,0 \\
\hline $\begin{array}{c}\text { Média } \\
\text { Total }\end{array}$ & - & $\mathbf{1 , 1 2}$ & $\mathbf{0 , 5 5}$ & $\mathbf{1 , 6} 7$ & - \\
\hline
\end{tabular}

*) Pulso avaliado: D = Direito; E = Esquerdo; D / E = Direito e Esquerdo.

Fonte: Dados da pesquisa 


\section{DISCUSSÃO}

A tenossinovite estenosante de De Quervain é uma afecção relacionada ao trabalho, principalmente às funções que desempenham movimentos repetitivos ${ }^{4,7^{3}}$. A incidência de doenças osteomusculares relacionadas ao trabalho vem aumentando e expandindo-se em todo o mundo, caracterizando uma doença limitante, interferindo na capacidade de realizar as atividades laborais e do cotidiano e gerando prejuízos pessoais e coletivos ${ }^{3,6}$. Nos Estados Unidos, ela é responsável por uma média de 20 bilhões de dólares destinados a reabilitação e tratamento profissional. Números semelhantes foram obtidos na Austrália, no ano de 2012, onde a mesma afecção foi responsável por até $43 \%$ do encargo anual destinado ao tratamento de doenças e lesões relacionadas ao trabalho ${ }^{10}$. Na Alemanha, os prejuízos resultam em dois milhões de dias de trabalhos perdidos ${ }^{6}$.

Tais números tendem a aumentar, levando em consideração o crescimento contínuo de dispositivos eletrônicos que requerem movimentos repetitivos das mãos e polegar, destacando a necessidade de identificar estratégias de gestão eficazes $^{12}$. O método terapêutico da tenossinovite De Quervain, utilizando infiltração de corticosteroide, é uma opção consagrada pela literatura e de ampla aplicabilidade na prática clínica, por tratar-se de procedimento com baixa complexidade, técnica simples com curva de aprendizado mais rápida, baixo ônus ao sistema de saúde e ao paciente, menor tempo de recuperação e retorno precoce às atividades laborais ${ }^{2,3,10,12}$. Cabe destacar, porém, a realização de estudos quantitativos se mostra fundamental para que se possa avaliar a extensão dos resultados e da eficácia desse método utilizado em pacientes acometidos pela tenossinovite De Quervain.

A tenossinovite de De Quervain foi descrita inicialmente, em 1895, por Fritz De Quervain, como uma entidade nosológica que aprisionava os tendões: Abdutor Longo do Polegar (ALP) e Extensor Curto do Polegar (ECP) no primeiro compartimento extensor do punho. Em 1893, já haviam relatos da afecção denominada "entorse da lavadeira", comumente causando dor e incapacidade funcional no punho e nas mãos das trabalhadoras braçais da época $^{4,6,10}$. Os tendões ALP e ECP deslizam num túnel osteoligamentar delimitado superiormente por fibras transversais dos ligamentos dorsais e inferiormente pelo processo estilóide do rádio. Esse túnel fibroso de baixa complacência mede cerca de 2 $\mathrm{cm}$ e forma um arcabouço rígido ${ }^{1,4,13,14}$.

É secundária a um processo inflamatório, ocasionando desproporção do conteúdo (ALP e ECP) em relação ao compartimento (túnel fibroso). Este processo pode ser agravado por atividades que requerem uso frequente das mãos, principalmente em desvio ulnar. Quanto maior o número de repetições e maior a força aplicada durante as atividades, maior será o atrito da bainha retinacular com os tendões, aumentando o edema e subseqüente o estreitamento do canal osteo-fibroso, e repercutindo em maior sintomatologia e piora dos déficits funcionais ${ }^{3,4,11}$.

A história de dor entre semanas a meses, localizada na topografia do primeiro compartimnto no lado dorsal do punho agravada com o movimento do polegar em desvio ulnar do punho leva à suspeição diagnóstica $^{5,11}$. Ao exame físico, evidenciase dor ou edema de $1 \mathrm{~cm}$ a $2 \mathrm{~cm}$ proximal ao estilóide radial, com piora a dos sintomas após flexão e adução do polegar na região palmar, com desvio ulnar forçado, uma manobra conhecida como Teste de Finkelstein, anteriormente denominada Teste de Eickhoff. O Teste de Mukart consiste em desvio ulnar do punho sem aprisionamento do polegar associado a dor em topografia do primeiro compartimento, e também pode ser utilizado ${ }^{11}$.

Exames de imagem auxiliam na elucidação diagnóstica nos casos atípicos ou diagnósticos diferencias. Ocasionalmente, radiografias podem evidenciar osteopenia localizada. Os exames de alta complexidade raramente são úteis para o diagnóstico dessa doença ${ }^{8,10,11,13}$.

Uma das opções terapêuticas descritas é a imobilização com tala gessada mantendo punho em extensão e polegar abduzido. Autores relatam recidiva após remoção da tala durante o reinício das atividades no membro acometido ${ }^{9,10,13}$.

A taxa de insucesso com o metodo de imobilização foi relativamente alta, iniciando a terapia combinada, adicionando a injeção de corticosteroíde no primeiro compartimento dorsal do punho. Os resultados foram promissores, tornando o método uma opção eficaz na forma de tratamento da doença de Quervain ${ }^{4,8}$.

O sucesso com injecção de corticosteróides tem sido relatado em $50 \%$ a $80 \%$ de pacientes, após uma a duas aplicações, particularmente eficaz em casos de manifestação aguda. As taxas de insucesso são mais elevadas em pacientes diabéticos ${ }^{2,3,8}$.

Em mulheres, a doença é autolimitada com resolução espontânea após término da gestação ou lactação, optando-se pelo tratamento conservador ${ }^{2,4,5}$. Comparações entre os métodos de imobilização com talas e injeção de corticoide demonstraram a resolução completa da dor e um resultado negativo no teste Finklestein, após a injeção de metilprednisolona em nove pacientes, ao mesmo tempo em que todos os pacientes do grupo com tala relataram dor durante o período de tratamento. Oito dos nove pacientes desse último grupo experimentaram resolução completa no período de duas a seis semanas após a interrupção da lactação².

Há diferentes marcas e apresentações de corticosteróides disponiveis no mercado, juntamente com diversas opções de solventes. A escolha do 
corticosteróide juntamente com o tipo de diluente interfere na duração e no tempo de ação da solução $^{9,13}$.

A técnica de infiltração também pode ser variável, conforme a preferência do profissional. Os tendões do primeiro compartimento dorsal podem facilmente ser palpados quando o paciente estende o polegar, auxiliando na identificação do ponto de infiltração. Uma agulha de calibre $\mathrm{n}^{\circ} 27$ é introduzida no túneo osteofibroso em topografia do primeiro compartimento extensor do punho e, nesse momento, sente-se uma resistência ao iniciar a injeção do medicamento, devendo-se, então, afastar lentamente a agulha do tendão, mantendo a pressão sobre $o$ êmbolo. A injeção pode ser melhorada ao se apalpar os tendões na topografia do estilóide radial, enquanto o paciente flexiona e estende a articulação do polegar. Uma segunda injeção pode ser realizada de quatro a seis semanas após a primeira aplicação, no entanto, as injeções repetidas não são indicados e podem ser prejudiciais aos tecidos locais ${ }^{8,11,13}$.

Embora a injeção local de corticosteróides seja um procedimento seguro, a possibilidade de efeitos colaterais existe e deve ser informada aos pacientes que serão submetidos ao tratamento. Os efeitos secundários, como o aumento transitório da dor e reações cutâneas locais, podem persistir por algum tempo após injeção e são, em grande maioria, transitórios ${ }^{15}$.

A falha no tratamento conservador é relatada entre $40 \%$ e $90 \%$ e pode estar associada às variações anatômicas no primeiro compartimento extensor do punho, tal como septos no primeiro compartimento, inserções anômalas dos tendões ou tendões supranumerários do abdutor longo do polegar ou extensor curto do polegar ${ }^{8,14-16}$.

O tratamento cirúrgico é indicado quando há falha no tratamento conservador. O procedimento é de curta duração, realizado com anestesia por infiltração local e uso de torniquete pneumático em torno do antebraço ${ }^{4}$. Para tanto, uma incisão de pele é feita transversalmente a $2 \mathrm{~cm}$ sobre o primeiro compartimento extensor e a cerca de $1 \mathrm{~cm}$ proximal ao processo estilóide. Deve haver cuidado para identificar e retrair delicadamente de um a três ramos sensitivos radiais que atravessam obliquamente o compartimento. Identifica-se o ligamento anular formando o teto do túnel ósseo-fibroso que deve ser incisado com um bisturi. A excisão completa de toda a bainha deve ser evitada porque, ocasionalmente, a mesma tem sido associada com dor palmar e subluxação dos tendões de pós-operatório. Os tendões ALP e ECP são identificados e expostos para fora do túnel para garantir a completa descompressão. Solicita-se ao paciente mover o polegar para demonstrar que o mesmo está livre e independente ${ }^{4,14}$.

Alguns autores consideram prudente o uso de tala aplicada com o punho em 20 graus de extensão, durante um período de 10 a 14 dias, embora seja raramente necessário. Depois disso, o paciente pode começar a retomar o uso do polegar e do punho, conforme tolerado. Recomenda-se evitar carregar peso ou realizar atividades mecânicas intensas nas primeiras quatro a seis semanas. A dor localizada pode persistir durante quatro a seis semanas no pósoperatório e, geralmente, responde bem à analgesia por via oral e às medidas analgésicas locais ${ }^{4,14}$.

\section{CONCLUSÃO}

A tenossinovite de De Quervain é uma afecção de grande importância na comunidade médica e na sociedade, principalmente ao se considerar as graves consequências e impactos na qualidade de vida e na saúde da população acometida.

Concluímos que o tratamento com infiltração de corticosteroides possui alta eficiência e grande viabilidade clínica e terapêutica, com impacto positivo na qualidade de vida dos pacientes, considerando o elevado índice de recuperação, retomada das atividades diárias, redução e/ou eliminação da dor no local afetado e retorno dos pacientes às suas funções laborais. Alguns pacientes persistiram com algum tipo de dor ou dificuldade ao realizar tarefas diárias, com predominância dos graus leves ou moderados de dor, enquanto a maioria dos pacientes avaliados apresentou recuperação total dos movimentos dos pulsos afetados e redução significativa e/ou eliminação completa da dor local.

Porém, reforçamos a necessidade de novos estudos envolvendo amostragens amplas e seguimento clínico prolongado para melhores avaliações a longo-prazo.

\section{REFERÊNCIAS}

1. Uribe WAJ, Buendia GPP, Rodriguez JMF, Vieira Filho JGC. Tenossinovites De Quervain: uma nova proposta no tratamento cirúrgico. Rev Bras Cir Plást. 2009;25(3):465-69.

2. Avci S, Ylmaz C, Sayli U. Comparison of nonsurgical treatment measures for de Quervain's disease of pregnancy and lactation. J Hand Surg Am. 2000;27(2):322-24.

3. Pensak MJ, Bayron J, Wolf JM. Current treatment of de Quervain tendinopathy. J Hand Surg Am. 2013;38(11):2247-49.

4. Pignataro MB, Praetzel RP. Tendinites e Tenossinovites. In: Pardini A, Freitas A. Cirurgia de mão: lesões não traumáticas. Rio de Janeiro: Medbook; 2008, p.509-20.

5. Alves MPT, Moraes Neto GP, Tzirulnik M. Avaliação clínico-ultra-sonográfica da tenossinovite estenosante de Quervain. Rev bras ortop. 2000;35(4):118-22.

6. Bonita R, Beaglehole R, Kjellström T. Epidemiologia básica. São Paulo: Santos; 2007. 
7. Diniz DRV, Mejia DPM. Ergonomia como fator de prevenção da doença tenossinovite estenosante de De Quervian no ambiente de trabalho. Disponível em: http://portalbiocursos.com.br/ohs/ data/docs/20/29_Ergonomia_como_fator_de_prev enYYo_da_doenYa_Tenossinovite_Estenosante_ de_De_Quervain_no_ambiente_de_trabalho.pdf. Acesso em: 16 jan. 2018.

8. Ansari MAQ. De Quervain's disease: a randomized prospective study evaluating the efficacy of steroid and conservative management. Int J Pharm Sci Invent. 2014;3(5):4-6.

9. Ashraf A, Hadianfard J. A comparison of the effect of acupuncture in treatment of De Quervain's disease with steroid injection. Ongoing study with unpublished results as of 2014. Eur J Orthop Surg Tr. 2014;26(4):157-68.

10. Ashraf MO, Devadoss VG. Systematic review and meta-analysis on steroid in-jection therapy for de Quervain's tenosynovitis in adults. Eur J Orthop Surg Tr. 2014;24(2):149-57.

11.Kutsumi K, Amadio PC, Zhao C, Zobitz ME, Tanaka T, An KN. Finkelstein's test: a biomechanical analysis. J Hand Surg Am. 2005;30(1):130-35.

12.McAuliffe JA. Tendon disorders of the hand and wrist. J Hand Surg Am. 2010;35(5):846-53.

13. Sawaizumi T, Nanno M, Ito H. De Quervain's disease: efficacy of intra-sheath triamcinolone injection. Int Orthop. 2007;31(2):265-68.

14. Shiraishi N, Matsumura G. Anatomical variations of the extensor pollicis brevis tendon and abductor pollicis longus tendon-relation to tenosynovectomy. Okajimas Folia Anat Jpn. 2005;82(1):25-9.

15. Harvey FJ, Harvey PM, Horsley MW. De Quervain's disease: surgical or nonsurgical treatment. J Hand Surg Am. 1990;15(1):83-7.

16. Tewari J, Mishra PR, Tripathy SK. Anatomical variation of abductor pollicis longus in Indian population: a cadaveric study. Ind $\mathrm{J}$ Orthop. 2015;49(5):549-53.

\section{CONFLITO DE INTERESSES}

Os autores declaram não haver conflitos de interesse.

AUTOR PARA CORRESPONDENCIA

\section{Bruno Moraes Vasconcelos}

brunomova@gmail.com
Submetido em 09/03/2019

Aceito em 19/03/2019 